\title{
Recurrence and fixed points of surface homeomorphisms
}

\author{
JOHN FRANKS \\ Department of Mathematics, Northwestern University, Evanston, IL 60201, USA
}

\begin{abstract}
We prove that if $f$ is a homeomorphism of the annulus which is homotopic to the identity and has a compact invariant chain transitive set $L$, then either $f$ has a fixed point or every point of $L$ moves uniformly in one direction: clockwise or counterclockwise. If $f$ is area-preserving, then the annulus itself is a chain transitive set, so, in the presence of a boundary twist condition, one obtains a fixed point. The same techniques apply to homeomorphisms of the torus $T^{2}$. In this setting we show that if $f$ is homotopic to the identity, preserves Lebesgue measure and has mean translation 0 , then it has at least one fixed point.
\end{abstract}

\section{Introduction}

One of the celebrated theorems of dynamical systems in the early part of this century was the so-called 'last geometric theorem of Poincaré'. While Poincaré conjectured this result and gave a partial proof, it was Birkhoff who first gave a complete proof. Roughly this result says that an area-preserving homeomorphism of the annulus which rotates one boundary component clockwise and the other counterclockwise possesses at least two fixed points.

The analogue of this result for the torus $T^{2}$ was conjectured by Arnold and proven by Conley and Zehnder [4]. It says that if an area-preserving diffeomorphism of $T^{2}$ is homotopic to the identity and has mean translation 0 (i.e. the mean rotation in both directions is zero), then that homeomorphism has at least three fixed points. Actually the conjecture of Arnold which Conley and Zehnder proved is more general and applies to symplectic diffeomorphisms of $T^{2 n}$, but our interest is in the surface case.

In this paper we investigate a different approach to these results in which a large role is played by recurrence. In $\$ 2$ we show (theorem 2.2 ) that if $f$ is a homeomorphism of the annulus with a compact invariant chain transitive set $\Lambda$, then either $f$ has a fixed point or every point of $\Lambda$ moves uniformly in one direction: clockwise or counterclockwise. If $f$ is area-preserving, then $A$ itself is a chain transitive set, so one nearly has a generalization of the last geometric theorem of Poincare (we obtain only one fixed point not two). It is not difficult to construct examples showing that one cannot conclude the existence of two fixed points with this weaker hypothesis.

This result has a number of interesting corollaries, which are 2.3, 2.4, 2.5 and 2.6 in the text. In particular we show that if $f$ is a homeomorphism of the annulus homotopic to the identity, then either every point has a well defined rotation number or $f$ has periodic points with infinitely many periods (see corollary 2.6). 
The proof of these results is based on a corollary of the Brouwer plane translation theorem (see $\S 1$ ).

It is natural to ask to what extent this result can be generalized to the torus. While a straightforward generalization is false, the techniques do lead to interesting results. In $\S 3$ we show that if $f: T^{n} \rightarrow T^{n}$ is a Lebesgue measure-preserving homeomorphism homotopic to the identity and has a lift $\tilde{f}: R^{n} \rightarrow R^{n}$ with mean translation vector zero, then every point of $R^{n}$ is chain recurrent under $\tilde{f}$ (see $\S 1$ for definitions). As a corollary one can show that if $n=2$ then the above hypotheses imply the existence of at least one fixed point for $f$. A final theorem (3.5) deals with an analogous result for a homeomorphism $f: T^{2} \rightarrow T^{2}$ which preserves an arbitrary ergodic Borel measure.

\section{Background and definitions}

We will make extensive use of the concept of chain recurrence which we now define (see [3] for more details).

Definition 1.1. If $f: X \rightarrow X$ is a homeomorphism of a metric space, an $\varepsilon$-chain from $x$ to $y$ is a sequence $x_{0}=x, x_{1}, x_{2}, \ldots, x_{n}=y, n \geq 1$, such that $d\left(f\left(x_{i}\right), x_{i+1}\right)<\varepsilon$. An invariant set $\Lambda$ is called chain transitive if for every $\varepsilon>0$ and every $x, y \in \Lambda$ there is an $\varepsilon$-chain from $x$ to $y$. A point $x \in X$ is chain recurrent if for every $\varepsilon>0$ there is an $\varepsilon$-chain from $x$ to $x$.

Proposition 1.2. Suppose $\Lambda$ is a closed connected invariant set in $X$ and every point of $\Lambda$ is chain recurrent. Then $\Lambda$ is chain transitive.

Proof. Given $x \in \Lambda$ and $\varepsilon>0$, let $A=\{y \in \Lambda \mid$ there is an $\varepsilon$-chain from $x$ to $y\}$. By definition $A$ is open in $\Lambda$. We show it is also closed. Suppose $y^{\prime}$ is in the closure of $A$. Then there is an $\varepsilon$-chain from $x$ to $y$ where $d\left(y, y^{\prime}\right)<\varepsilon / 2$. Since $y$ is chain recurrent, there is an $(\varepsilon / 2)$-chain from $y$ to $y$ and hence an $\varepsilon$-chain from $y$ to $y^{\prime}$. Following the chain from $x$ to $y$ by one from $y$ to $y^{\prime}$ gives the desired result.

An important ingredient of our subsequent results, and in fact the ultimate theorem we appeal to for the existence of fixed points, is a corollary of a result of Brouwer on orientation-preserving homeomorphisms of the plane. While the full theory of Brouwer is quite deep, the result we need is a corollary of an easy part of this theory. A modern treatment quite adequate for our needs can be found in [2]. The result we need is the following:

THEOREM 1.3. Iff: $R^{2} \rightarrow R^{2}$ is an orientation-preserving homeomorphism with a periodic point $p$, then $f$ posssesses a fixed point.

Proof. If $p$ is not itself fixed, let $a=\sup \left\{r \mid B_{r}(p) \cap f\left(B_{r}(p)\right)=\varnothing\right\}$, where $B_{r}(p)$ is the ball of radius $r$ centred at $p$. Let $x \in \partial B_{a}(p) \cap \partial f\left(B_{a}(p)\right)$ and choose an arc $\gamma$ from $f^{-1}(x) \in \partial B_{a}(p)$ to $x$ which, except for its endpoints, lies in the interior of $B_{a}(p)$ and which passes through $p$.

The main result of [2] (theorem 1) shows that either $f^{n}(\gamma) \cap \gamma=\varnothing$ for all $n \geq 2$ or $f$ has a fixed point. Since $\gamma$ contains the periodic point $p$, the first option is clearly impossible and we must have a fixed point of $f$. 


\section{Homeomorphisms of the annulus}

LEMMA 2.1. Suppose $f: M^{2} \rightarrow M^{2}$ is a homeomorphism of a compact surface whose universal covering space $\tilde{M}$ is either $R^{2}$ or $R \times I$. If $\tilde{f}: \tilde{M} \rightarrow \tilde{M}$ is a lift of $f$ which has a chain recurrent point, then $\tilde{f}$ has a fixed point (and hence so does $f$ ).

Proof. Suppose to the contrary that $\tilde{f}$ has no fixed point. Then since $M$ is compact there exists $\delta>0$ such that $d(\tilde{f}(x), x)>\delta$ for all $x \in \tilde{M}$. Hence no perturbation $\tilde{\mathbf{g}}$ of $\tilde{f}$ such that $d(\tilde{g}(x), \tilde{f}(x))<\delta / 2$, for all $x$, can have a fixed point.

We will use the corollary of the Brouwer theorem (1.3) to show that there is a $\tilde{\mathbf{g}}$ arbitrarily close to $\tilde{f}$ which does have a fixed point and hence complete the proof by contradiction.

Let $x_{0}, x_{1}, \ldots, x_{n+1}=x_{0}$ be a $(\delta / 4)$-chain for $\tilde{f}$. A result of [6] (theorem 4) says that it is possible to find disjoint polygonal arcs $\gamma_{i}$ joining $f\left(x_{i}\right)$ to $x_{i+1}$ (and $f\left(x_{n}\right)$ to $x_{0}$ in the case of $\gamma_{n}$ ) with each $\gamma_{i}$ having diameter $<\delta / 3$. We can then construct a homeomorphism $h$ of $\tilde{M}$ supported on small neighbourhoods of the $\gamma_{i}$ such that $h\left(f\left(x_{i}\right)\right)=x_{i+1}\left(\right.$ and $\left.h\left(f\left(x_{n}\right)\right)=x_{0}\right)$ and with the property that

$$
d(h(x), x)<\delta / 2 \quad \text { for all } x \in \tilde{M} \text {. }
$$

Let $\tilde{g}(x)=h(\tilde{f}(x))$. It follows from the remarks above that $d(\tilde{f}(x), \tilde{g}(x))<\delta / 2$ for all $x \in \tilde{M}$. Also $\tilde{g}^{n}\left(x_{0}\right)=x_{0}$, so by theorem 1.3 there is a fixed point for $\tilde{g}$.

THEOREM 2.2. Suppose $f: A \rightarrow A$ is a homeomorphism of the annulus which is homotopic to the identity map and $\Lambda \subset A$ is a chain transitive compact invariant set. Let $\tilde{f}: R \times I \rightarrow$ $R \times I$ be a lift of $f$; then one of the following is true:

(1) the lift $\tilde{f}$ has a fixed point, or

(2) for all $x \in \Pi^{-1}(\Lambda)$

$$
\lim _{n \rightarrow \infty}\left(\tilde{f}^{n}(x)-x\right)_{1}=\infty,
$$

where $\Pi: R \times I \rightarrow A$ is the covering projection and ( ) denotes the first component, or

(3) for all $x \in \Pi^{-1}(\Lambda)$

$$
\lim _{n \rightarrow \infty}\left(\tilde{f}^{n}(x)-x\right)_{1}=-\infty
$$

In cases (2) and (3) the limits are uniform in $x$.

Proof. In view of lemma 2.1, to prove (1) it suffices to show the existence of a chain recurrent point for $\tilde{f}$. Our strategy is to show that if (2) and (3) are false then there is a chain recurrent point and hence (1) holds.

We want first to show that given $M>0$ either $\tilde{f}$ has a fixed point, (2) holds, (3) holds, or there are points $y_{1}, y_{2} \in \Pi^{-1}(\Lambda)$ such that

$$
\left(\tilde{f}^{p}\left(y_{1}\right)-y_{1}\right)_{1}>M \quad \text { for some } p>0
$$

and

$$
\left(\tilde{f}^{q}\left(y_{2}\right)-y_{2}\right)_{1}<-M \quad \text { for some } q>0 .
$$

Suppose for some $M$ that one of these latter inequalities fails (say the second, the other case being similar). Then for all $y \in \Pi^{-1}(\Lambda)$ and all $n>0,\left(\tilde{f}^{n}(y)-y\right)_{1} \geq-M$. 
If for any $x \in \Pi^{-1}(\Lambda)$ the set of numbers $\left(\tilde{f}^{n}(x)-x\right)_{1}$ is bounded, then $\left\{f^{n}(x)\right\}$ has a limit point which is chain recurrent and hence $\tilde{f}$ has a fixed point.

If we suppose this is not the case, then we know that for any $x \in \Pi^{-1}(\Lambda)$ the set $\left(\tilde{f}^{n}(x)-x\right)_{1}$ is unbounded but bounded below by $-M$. That is, for every $x \in \Pi^{-1}(\Lambda)$ powers of $\tilde{f}$ can move $x$ arbitrarily far to the right, but can move it at most a distance $M$ to the left. Hence $\lim _{n \rightarrow \infty}\left(\tilde{f}^{n}(x)\right)_{1}=\infty$ for all $x \in \Pi^{-1}(\Lambda)$. The fact that this limit is uniform in $x$ follows easily from the fact that $\tilde{f}$ commutes with the deck translation $x \mapsto x+(1,0)$ and the compactness of $\Pi^{-1}(\Lambda) \cap([0,1] \times I)$. Thus we have shown that either $\tilde{f}$ has a fixed point, (2) holds, (3) holds, or for any given $M>0$ there are points $y_{1}, y_{2} \in \Pi^{-1}(\Lambda)$ such that

$$
\left(\tilde{f}^{p}\left(y_{1}\right)-y_{1}\right)_{1}>M \quad \text { for some } p
$$

and

$$
\left(\tilde{f}^{q}\left(y_{2}\right)-y_{2}\right)_{1}<-M \quad \text { for some } q .
$$

We now show that the existence of such $y_{1}$ and $y_{2}$ (depending on $M$ ) also implies the existence of a fixed point for $\tilde{f}$.

We are now prepared to prove the existence of a chain recurrent point for $\tilde{f}$. Let $z_{1}, z_{2} \in \Pi^{-1}(\Lambda)$ satisfy $\left|\left(z_{1}-z_{2}\right)_{1}\right|<1$. Given any $\varepsilon>0$ there is an $\varepsilon$-chain from $\pi\left(z_{1}\right)$ to $\pi\left(z_{2}\right)$ whose length depends only on $\varepsilon$ (not on $z_{1}$ or $z_{2}$ ) since $\Lambda$ is compact. If we lift this $\varepsilon$-chain to $R \times I$ starting at $z_{1}$, we obtain an $\varepsilon$-chain from $z_{1}$ to $z_{2}+(n, 0)$ for some $n$. Since the length of the chain is bounded and $\tilde{f}$ moves points a bounded distance, there is a bound $N$ depending only on $\varepsilon$ such that $|n|<N$.

We now choose our points $y_{1}$ and $y_{2}$ as above with $M=4 N$. Starting now with any $z \in \Pi^{-1}(\Lambda)$, we form an $\varepsilon$-chain from $z$ to $y_{1}^{\prime}=y_{1}+(k, 0)$ by lifting an $\varepsilon$-chain on $A$. The remarks above show we can assume $\left|\left(z-y_{1}^{\prime}\right)_{1}\right|<N$. We continue this $\varepsilon$-chain by adding $\tilde{f}^{j}\left(y_{1}^{\prime}\right), 1 \leq j \leq p$. Note that

$$
\left(\tilde{f}^{p}\left(y_{1}^{\prime}\right)-y_{1}^{\prime}\right)_{1}>4 N \text {. }
$$

Finally, by lifting a chain on $\boldsymbol{A}$ again, we continue this chain to go from $\tilde{f}^{p}\left(y_{1}^{\prime}\right)$ to $z^{\prime}=z+(r, 0)$ and once again we have $\left|\left(\tilde{f}^{p}\left(y_{1}^{\prime}\right)-z^{\prime}\right)_{1}\right|<N$. Thus we have constructed an $\varepsilon$-chain from $z$ to $z^{\prime}=z+(r, 0)$ and our estimates imply $r>2 N$.

A completely analogous argument shows the existence of an $\varepsilon$-chain from $z$ to $z^{\prime \prime}=z-(s, 0)$ with $-s<-2 N$. We can, of course, translate this $\varepsilon$-chain by $(n, 0)$ for any integer $n$ and obtain another one. Hence it is possible to juxtapose $s$ of the originals to get an $\varepsilon$-chain from $z$ to $z+(r s, 0)$. Following this with $r$ copies of the other going from $z+(r s, 0)$ to $z$ gives an $\varepsilon$-chain from $z$ to $z$. Since $z \in \Pi^{-1}(\Lambda)$ was arbitrary, as was $\varepsilon>0$, we have shown that every point of $\Pi^{-1}(\Lambda)$ is chain recurrent for $\tilde{f}$ if neither (2) nor (3) holds. Together with lemma 2.1 this proves the theorem.

Corollary 2.3. Suppose $f: A \rightarrow A$ is a chain transitive homeomorphism homotopic to the identity. If $\tilde{f}: R \times I \rightarrow R \times I$ is a lift of $f$, then either:

(1) $\tilde{f}$ has a fixed point, or

(2) $\lim _{n \rightarrow \infty}\left(\tilde{f}^{n}(x)-x\right)_{1}=\infty$ for all $x$, or

(3) $\lim _{n \rightarrow \infty}\left(\tilde{f}^{n}(x)-x\right)_{1}=-\infty$ for all $x$. 
This is immediate from theorem 2.2 taking $\Lambda=A$. Note that this is closely related to the so-called 'last geometric theorem of Poincaré'. While we obtain as conclusion only the existence of one fixed point (not two), our hypothesis of chain transitivity is much weaker than the hypothesis of area-preserving.

It is not difficult to show one cannot improve this result to two fixed points, since there are easy examples of chain transitive homeomorphisms of $A$ with a single fixed point.

COROLlary 2.4. Suppose that $\tilde{f}$ and $\Lambda$ are as in theorem 2.2 and $x, y \in \Pi^{-1}(\Lambda)$. If

$$
\lim \inf \frac{1}{n}\left(\tilde{f}^{n}(x)-x\right)_{1} \leq p / q \leq \lim \sup \frac{1}{n}\left(\tilde{f}^{n}(y)-y\right)_{1},
$$

then $f$ has a periodic point with rotation number $p / q$.

Proof. Let $T: R \times I \rightarrow R \times I$ be the covering translation $T(x)=x+(1,0)$. If $x_{0}$ is a fixed point of $T^{-p} \circ \tilde{f}^{q}$, then $\pi\left(x_{0}\right)$ is a periodic point of $f$ with rotation number $p / q$. Since $\tilde{g}=T^{-p} \circ \tilde{f}^{q}$ is a lift of $f^{q}$, we can apply theorem 2.2 to it. We first observe that

$$
\lim \inf \frac{1}{n}\left(\tilde{g}^{n}(x)-x\right)_{1} \leq 0 \leq \lim \sup \frac{1}{n}\left(\tilde{g}^{n}(y)-y\right)_{1} .
$$

Note that if $\lim \left(\tilde{g}^{n}(x)-x\right)_{1}=\infty$, uniformly in $x$, then for some $N>0,\left(\tilde{g}^{N}(x)-x\right)_{1}>$ 1 for all $x \in \Pi^{-1}(\Lambda)$. A telescoping sum then shows that

$$
\lim \inf \frac{1}{n}\left(\tilde{g}^{n}(x)-x\right)_{1} \geq \frac{1}{N},
$$

so (2) of theorem 2.2 is not possible. Likewise (3) of theorem 2.2 is excluded. Hence $\tilde{g}=T^{-p} \circ \tilde{f}^{q}$ has a fixed point.

The following result has been proved independently by Handel [5] by quite different methods.

Corollary 2.5. Suppose $f: A \rightarrow A$ is a homeomorphism homotopic to the identity. If $\tilde{f}: R \times I \rightarrow R \times I$ is a lift of $f$ and for some $x \in R \times I$

$$
\lim \inf \frac{1}{n}\left(\tilde{f}^{n}(x)-x\right)_{1} \leq p / q \leq \lim \sup \frac{1}{n}\left(\tilde{f}^{n}(x)-x\right)_{1},
$$

then $f$ has a periodic point with rotation number $p / q$.

Proof. Let $\Lambda=\omega(\Pi(x))$, the $\omega$-limit set of $\Pi(x)$. Then $\Lambda$ is a compact invariant chain transitive set. Once again we consider $f^{q}$ and its lift $\tilde{g}=T^{-p} \circ \tilde{f}^{q}$. By theorem 2.2 we know that if $\tilde{g}$ has no fixed point then

$$
\lim _{n \rightarrow \infty}\left(\tilde{g}^{n}(y)-y\right)_{1}= \pm \infty \text { uniformly in } y \in \Pi^{-1}(\Lambda) \text {. }
$$

We suppose it is $+\infty$, the other case being similar. Then there exists $N>0$ such that

$$
\left(\tilde{g}^{N}(y)-y\right)_{1}>2 \quad \text { for all } y \in \Pi^{-1}(\Lambda) \text {. }
$$

Since $f^{k}(\Pi(x))$ limits on the compact set $\Lambda$, it follows that for $n$ sufficiently large

$$
\left(\tilde{g}^{N+n}(x)-\tilde{g}^{n}(x)\right)_{1}>1
$$


and hence

$$
\left(\tilde{g}^{k N+n}(x)-\tilde{g}^{n}(x)\right)_{1}>k
$$

This clearly contradicts the assumption that

$$
\lim \inf \frac{1}{n}\left(\tilde{g}^{n}(x)-x\right)_{1} \leq 0 .
$$

The case that $\lim \left(\tilde{g}^{n}(y)-y\right)_{1}=-\infty$ is handled similarly, so we conclude that $\tilde{g}$ has a fixed point.

The following result is then an immediate corollary.

Corollary 2.6. Suppose $f: A \rightarrow A$ is a homeomorphism homotopic to the identity and that among all periodic points of $f$ there are only finitely many periods. Then every point of $A$ has a rotation number. If $f$ is chain transitive, then all these rotation numbers are equal.

\section{Homeomorphisms of $T^{n}$}

Definition 3.1. Let $f: T^{n} \rightarrow T^{n}$ be a homeomorphism homotopic to the identity which preserves a Borel measure $\mu$, and let $\tilde{f}: R^{n} \rightarrow R^{n}$ be a lift. We define the mean translation vector of $\tilde{f}$ with respect to $\mu$ to be

$$
\int_{\Omega}(\tilde{f}(x)-x) \mu,
$$

where $\Omega$ is a fundamental domain.

THEOREM 3.2. Suppose $f: T^{n} \rightarrow T^{n}$ is a homeomorphism which preserves Lebesgue measure and is homotopic to the identity. If $\tilde{f}: R^{n} \rightarrow R^{n}$ is a lift with mean translation vector zero, then every point of $R^{n}$ is chain recurrent for $\tilde{f}$.

Proof. Let $e_{1}, e_{2}, \ldots, e_{n}$ be the standard basis of $R^{n}$ and let $e_{n+1}=$ $(1 / \sqrt{n})(-1,-1, \ldots,-1)$, so the vectors $\left\{e_{1}, \ldots, e_{n+1}\right\}$ have 0 in the interior of their convex hull. Given $\varepsilon>0$ and $x \in R^{n}$ we wish to find an $\varepsilon$-chain from $x$ to $x+\nu_{i}$, where $\nu_{i}$ is an integer vector very close in direction to $e_{i}$. More precisely we require $\nu_{i} /\left|\nu_{i}\right|$ to be sufficiently close to $e_{i}$ that 0 is in the convex hull of $\left\{\nu_{i} /\left|\nu_{i}\right|\right\}$.

We postpone the proof of the existence of $\left\{\nu_{i}\right\}$ and complete the rest of the proof. Since 0 is in the convex hull of $\left\{\nu_{i} /\left|\nu_{i}\right|\right\}$, we can find positive integers $a_{i}$ such that

$$
\sum_{i=1}^{n+1} a_{i} \nu_{i}=0 \text {. }
$$

The $\varepsilon$-chains from $x$ to $x+\nu_{i}$ can be translated by any integer vector and will still remain $\varepsilon$-chains. Hence we can juxtapose $a_{1}$ of the $\varepsilon$-chains from $x$ to $x+\nu_{1}$, placing them one after the other, and then follow this by $a_{2}$ of the $\varepsilon$-chains parallel to $\nu_{2}$ and then $a_{3}$ of those parallel to $\nu_{3}$, etc. Since $\sum a_{i} \nu_{i}=0$, the result will be an $\varepsilon$-chain from $x$ to $x$ as desired.

Hence we need only show the existence of the integer vectors $\nu_{i}$ and their corresponding $\varepsilon$-chains. We consider the homeomorphism $\tilde{h}_{i}: R^{n} \rightarrow R^{n}$ given by $\tilde{h}_{i}(x)=\tilde{f}(x)+(\varepsilon / 2) e_{i}$. Obviously it has mean translation vector $(\varepsilon / 2) e_{i}$ and is the 
lift of a homeomorphism $h_{i}$ on $T^{n}$ which preserves Lebesgue measure. By the Oxtoby-Ulam theorem [7] there is a homeomorphism $g_{i}: T^{n} \rightarrow T^{n}$ which preserves Lebesgue measure and is ergodic and which is as close as we wish to $h_{i}$ in the $C^{0}$ topology. We choose the $g_{i}$ sufficiently close that their lifts $\tilde{g}_{i}: R^{n} \rightarrow R^{n}$ have mean translation vectors $\alpha_{i}$ close enough to $(\varepsilon / 2) e_{i}$ that 0 is in the interior of their convex hull, and so that $\left|\tilde{g}_{i}(x)-\tilde{h}_{i}(x)\right|<\varepsilon / 4$ for all $x$.

Now for all $x \in R^{n}$ except a set of measure zero, $\Pi(x) \in T^{n}$ will be recurrent for every $\boldsymbol{g}_{i}$.

Let $\phi_{i}(x)=\tilde{g}_{i}(x)-x$; then

$$
\alpha_{i}=\int_{\Omega} \phi_{i}(x) d x=\lim _{n \rightarrow \infty} \frac{1}{N} \sum_{n=0}^{N} \phi_{i}\left(\tilde{g}_{i}^{n}\left(x_{0}\right)\right)
$$

for almost all $x_{0}$ by the ergodic theorem. But

$$
\left.\frac{1}{N} \sum \phi_{i}\left(\tilde{g}_{i}^{n}\left(x_{0}\right)\right)=\frac{1}{N}\left(\tilde{g}_{i}^{N+1}\left(x_{0}\right)-x_{0}\right)\right)
$$

so

$$
\lim _{n \rightarrow \infty} \frac{1}{n}\left(\tilde{g}_{i}^{n}\left(x_{0}\right)-x_{0}\right)=\alpha_{i} .
$$

Since we can choose $x_{0}$ so $\Pi\left(x_{0}\right)$ is recurrent for $g_{i}$, we can then choose $n$ so that $\tilde{g}_{i}^{n}\left(x_{0}\right)=x_{0}+\nu_{i}+\rho_{i}$, where $\nu_{i}$ is an integer vector, $\left|\rho_{i}\right|<\varepsilon / 4$ and $n$ is sufficiently large that $\nu_{i} / n$ is very close to $\alpha_{i}$. We of course have a different $n$, say $n_{i}$, for each $i$. But if the $\nu_{i} / n_{i}$ are sufficiently close to the $\alpha_{i}$, then 0 will still be in the interior of the convex hull of $\left\{\nu_{i} /\left|\nu_{i}\right|\right\}$.

It remains only to show there is an $\varepsilon$-chain for $\tilde{f}$ from $x_{0}$ to $x_{0}+\nu_{i}$, but since $\tilde{g}_{i}$ is an $(\varepsilon / 2)$-approximation to $\tilde{f}$, its orbit $x_{0}, \tilde{g}\left(x_{0}\right), \tilde{g}^{2}\left(x_{0}\right), \ldots, \tilde{g}^{n}\left(x_{0}\right)$ is an $(\varepsilon / 2)$-chain for $\tilde{f}$. Making the last little $(\varepsilon / 4)$-jump from $\tilde{g}^{n}\left(x_{0}\right)$ to $x_{0}+\nu_{i}$ gives an $\varepsilon$-chain for $\tilde{f}$. Since the point $x_{0}$ was any point in a set of full measure in $R^{n}$, we conclude that for any $x \in R^{n}$ and any $\varepsilon>0$ there is an $\varepsilon$-chain from $x$ to $x$.

COROLlARY 3.3. If $f: T^{2} \rightarrow T^{2}$ is a homeomorphism homotopic to the identity which preserves Lebesgue measure and has a lift with mean translation vector 0 , then $f$ has a fixed point.

This is immediate from theorem 3.2 and lemma 2.1. We note that this result also follows from the much stronger results of Conley and Zehnder [4], who show that if $f$ is a diffeomorphism it has three fixed points. The question of whether a homeomorphism must have three fixed points is apparently open.

The following example was shown to me by Michel Herman.

Example 3.4. Let $f: T^{3} \rightarrow T^{3}$ be given by $f(x, y, z)=(x+\cos z, y+\sin z, z)$, where each coordinate is taken mod 1 . Then there is a lift to $R^{3}, \tilde{f}$, which is defined by the same formula. It is easy to check that $\tilde{f}$ preserves Lebesgue measure and has mean translation vector zero. On the other hand each point $p \in R^{3}$ possesses a neighbourhood $U_{p}$ such that $\tilde{f}^{n}\left(U_{p}\right)$ tends to $\infty$ as $n \rightarrow \infty$. In particular $\tilde{f}$ has an empty non-wandering set. In light of this, our result (3.2) which says every point of $R^{3}$ is chain recurrent under $\tilde{f}$ seems unlikely to be amenable to strengthening. 
It is interesting to ask to what extent the above results depend on having Lebesgue measure invariant. One can easily construct examples which are chain transitive homeomorphisms of $T^{2}$ and have no fixed points.

One can however obtain a result for an arbitrary invariant Borel measure provided it is ergodic.

THEOREM 3.5. Suppose $f: T^{2} \rightarrow T^{2}$ is a homeomorphism homotopic to the identity which preserves a Borel measure $\mu$ and is ergodic. If $f$ has a lift whose mean translation vector with respect to $\mu$ is zero, then $f$ has a fixed point.

Proof. By lemma 2.1 it is sufficient to find a chain recurrent point for the lift $\tilde{f}: R^{2} \rightarrow R^{2}$ with mean translation vector 0 . As in the proof of theorem 3.2, we need only find $\varepsilon$-chains from a point $x \in R^{2}$ to points $\left\{x+\nu_{i}\right\}$, where $\left\{\nu_{i}\right\}$ are integer vectors with 0 in their convex hull, because by piecing these $\varepsilon$-chains together we can find an $\varepsilon$-chain from $x$ to $x$.

Let $\Lambda=\operatorname{supp}(\mu)$ and choose $x_{0} \in \Pi^{-1}(\Lambda)$ such that $\Pi\left(x_{0}\right)$ is recurrent. Given $\varepsilon>0$ let $V=\left\{\nu \in Z^{2} \mid\right.$ there exists an $\varepsilon$-chain from $x_{0}$ to $\left.x_{0}+\nu\right\}$. We note that there are $\varepsilon$-chains for $f$ on $T^{2}$ from $\Pi\left(x_{0}\right)$ to $\Pi\left(x_{0}\right)$, and lifting them to $R^{2}$ shows that $V$ is not empty.

We need only show that 0 is in the convex hull of $V$ (and hence in fact in $V$ ). If this were not the case then $V$ would be contained in some half-space of $R^{2}$, and we will show this to be impossible.

Suppose without loss of generality that $V \subset H=\{(x, y) \mid y \geq 0\}$ and let ()$_{1}$ and ( ) $)_{2}$ denote the first and second components of points in $R^{2}$. Let $\phi(z)=\tilde{f}(z)-z$ so $\phi$ is a periodic function on $R^{2}$ and can be thought of as a function on $T^{2}$. Since $\tilde{f}$ has mean translation 0 , we have

$$
\int_{T^{2}} \phi(z) \mu=0 .
$$

Thus since $f$ is ergodic,

$$
\lim _{n \rightarrow \infty} \frac{1}{n} \sum_{n=0}^{\infty} \phi\left(f^{n}(z)\right)=0
$$

for almost all $z$.

A result of [1] says that if we use a real-valued function $g: T^{2} \rightarrow R$ with $\mu$ mean 0 (instead of the vector-valued $\phi$ ), we will have

$$
\left|\sum_{n=0}^{N} g\left(f^{n}(z)\right)\right|<\varepsilon
$$

for almost all $z$ and infinitely many $N>0$.

We will apply $\left(^{*}\right)$ in the case when $g(z)=(\phi(z))_{1}$ and when $g(z)=(\phi(z))_{2}$. We choose $z_{0}$ which is recurrent for $f$ and for which $\left(^{*}\right)$ is valid for both choices of $g$. Then let $x_{0} \in \Pi^{-1}\left(z_{0}\right)$.

Now there exists an increasing sequence $\{K(i)\}$ such that

$$
\left|\sum_{n=0}^{K(i)}\left(\phi\left(f^{n}\left(z_{0}\right)\right)\right)_{1}\right|<\varepsilon \quad \text { for all } i
$$


Hence

$$
\left|\left(\tilde{f}^{K(i)+1}\left(x_{0}\right)-x_{0}\right)_{1}\right|<\varepsilon \quad \text { for all } i .
$$

Let $\omega_{i}=\tilde{f}^{K(i)+1}\left(x_{0}\right)$. Then the sequence $\left\{\omega_{i}\right\}$ must be unbounded in $R^{2}$ since otherwise it would have a limit point which would be recurrent. It follows that $\left\{\left(\omega_{i}\right)_{2}\right\}$ is unbounded and positive.

Now applying $\left(^{*}\right)$ to $(\phi(z))_{1}$, we conclude that there is an increasing sequence $\{N(j)\}$ such that

$$
\left|\sum_{n=0}^{N(j)}\left(\phi\left(f^{n}\left(z_{0}\right)\right)\right)_{2}\right|<\varepsilon \quad \text { for all } j
$$

and hence

$$
\left|\left(\tilde{f}^{N(j)+1}\left(x_{0}\right)-x_{0}\right)_{2}\right|<\varepsilon .
$$

Let $u_{j}=\tilde{f}^{N(j)+1}\left(x_{0}\right)$; then $\left\{\left|\left(u_{j}\right)_{1}\right|\right\}$ is unbounded but $\left|\left(u_{j}\right)_{2}\right|<\varepsilon$ for all $j$.

Now for each $i$ choose a $j(i)$ such that $N(j(i))>K(i)$. We then have $\tilde{f}^{M(i)}\left(\omega_{i}\right)=$ $u_{i}^{\prime}$, where $M(i)=N(j(i))-K(i)$ and $u_{i}^{\prime}=u_{j(i)}$. Clearly $\left\{\left(u_{i}^{\prime}-\omega_{i}\right)_{2}\right\}$ are unbounded and negative and there is an $\varepsilon$-chain (in fact an orbit segment) from $\omega_{i}$ to $u_{i}^{\prime}$.

As in the proof of theorem 3.2, we can lift $\varepsilon$-chains on $T^{2}$ to $R^{2}$ and obtain an $\varepsilon$-chain from $x_{0}(i)$ to $\omega_{i}$, where $x_{0}(i)$ is an integer translate of $x_{0}$ and $\left|x_{0}(i)-\omega_{i}\right|<C$, where $C$ is a constant $>0$ depending on $\varepsilon$ but not $i$. Likewise we can find an $\varepsilon$-chain from $u_{i}^{\prime}$ to $x_{0}^{\prime}(i)$, where $x_{0}^{\prime}(i)$ is an integer translate of $x_{0}$ and $\left|x_{0}^{\prime}(i)-u_{i}^{\prime}\right|<C$.

Choosing $i$ so that $\left(\omega_{i}-u_{i}^{\prime}\right)_{2}>3 C$ and piecing together the $\varepsilon$-chains we have constructed, we get an $\varepsilon$-chain from $x_{0}(i)$ to $x_{0}^{\prime}(i)$ with $\left(x_{0}(i)-x_{0}^{\prime}(i)\right)_{2}>C$. This implies that there is a $\nu^{\prime} \in V$ with $\left(\nu^{\prime}\right)_{2}<-C$, contradicting the assumption that $V$ was contained in a half-plane.

Acknowledgement. This research was supported in part by NSF Grant MCS 83-01067.

\section{REFERENCES}

[1] G. Atkinson. Recurrence of cocycles and random walks. J. Lond. Math. Soc. 13 (1976), 486-488.

[2] M. Brown. A new proof of Brouwer's lemma on translation arcs. Houston J. Math. 10 (1984), 35-41.

[3] C. Conley. Isolated Invariant Sets and the Morse Index. CBMS Regional Conf. Series in Math. 38. AMS, Providence, RI (1978).

[4] C. Conley \& E. Zehnder. The Birkhoff-Lewis fixed point theorem and a conjecture of Arnold. Invent. Math. 73 (1983), 33-49.

[5] M. Handel. To appear.

[6] J. Oxtoby. Diameters of arcs and the gerrymandering problem. Amer. Math. Monthly 84 (1977), $155-162$.

[7] J. Oxtoby \& S. Ulam. Measure preserving homeomorphisms and metrical transitivity. Ann. Math. 42 (1941), 874-920. 